\title{
Regional Urban Area Extraction Using MODIS data and DMSP/OLS data
}

\author{
YingLV \\ School of Geography \\ Beijing Normal University \\ Beijing, China \\ Lvying.2010@163.com
}

\author{
HuipingLIU \\ School of Geography \\ Beijing Normal University \\ Beijing, China \\ hpliu@bnu.edu.cn
}

\begin{abstract}
Regional urban extraction is an essential process in analyzing urban agglomeration based on GIS and RS.Urban agglomeration will become the main form of urbanization of China in the future .In this paper, we present results from efforts to map urban agglomeration built-up land at $500 \mathrm{~m}$ spatial resolution using MODIS and DMSP/OLS data. This research builds a new building index (NBI) to map regional urban based on MODIS and DMSP/OLS data. Then we verify the validity of the new building index using stratified random accuracy assessment method compares with NDBI and IBI.The result shows that new building index has an overall accuracy of $87.25 \%$ $(k=0.6537), 87.50 \%(k=0.6713)$ and $90.50 \%(k=0.8022)$ at the pixel level and a high level of agreement at the city scale $\left(R^{2}>0.9\right)$ in Beijing-tianjin-hebei, Yangtze River Delta and Pearl River Delta urban agglomeration.
\end{abstract}

\section{Keywords - urban extraction,NBI,MODIS,DMSP}

\section{INTRODUCTION}

Urban agglomeration will become the main form of urbanization of China in the future. Regional urban extraction is an essential process in analyzing urban agglomeration based on GIS and RS. Extracting regional urban area accurately that has significance for understanding the spatial structure of urban agglomerations.

Many researches have made use of remote image to extract urban in global or regional. The usual method is decision tree classification to extract regional urban area based on MODIS data ${ }^{[1-3]}$. Much prior knowledge is needed for extracting building land accurately at low resolution. The Normalized Difference Build-up Index (NDBI) is commonly used to extraction of built-up land based on TM image ${ }^{[4-6]}$. Other indexes are using to assist extraction at high resolution. In this study build a new building index based on MODIS and DMSP data. The stable night light data is prior knowledge for new building index that extract regional urban area accurately and simply.

This paper building new building index through observing standard spectral from MOD09A1 and DMSP/OLS constitute the correction factor for new building index. Then we verify the validity of the new building index inBeijing-tianjin-hebei, Yangtze River Delta and Pearl River Delta urban agglomeration where the new building index is compared with NDBI and IBI.Finally, the accuracy assessment method is confusion matrix and Kappa coefficientbased on stratifiedrandom sampling. The reference data is Google Earth image at $30 \mathrm{~m}$ spatial resolution.

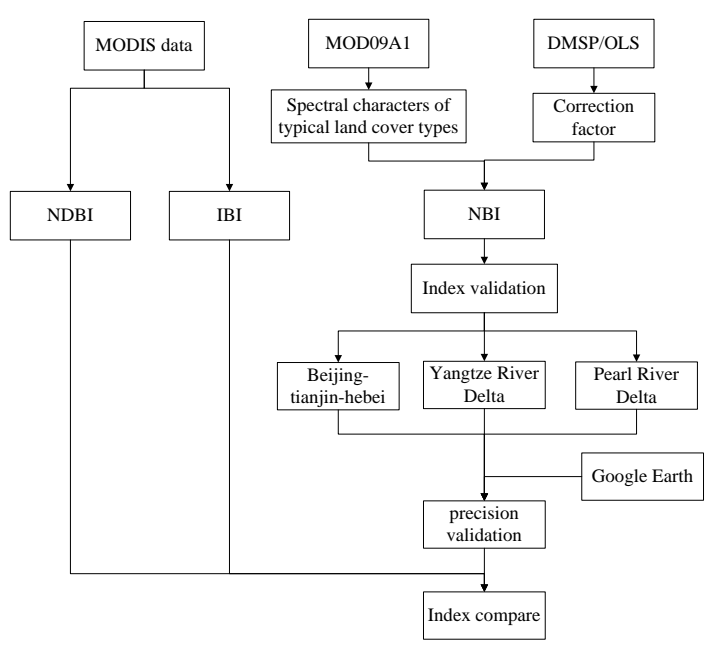

Fig.1 the flowchart of the paper

\section{METHODOLOGY}

NDBI (Normalized Difference Built-up Index) is an effective index in mapping general built-up areas based on TM image. Built-up areas and barren land experience a drastic increment from band 4 to band 5 while vegetation has a slightly larger or smaller DN value on band 5 than on band $4^{[4]}$. It is the theory of NDBI, as expressed in Eq. 1.

$$
\text { NDBI }=(\text { TM5 }- \text { TM4 }) /(T M 5+T M 4)(1)
$$

The central wavelength of TM4 and TM5 are 0.83 and 1.67 $\mathrm{nm}$.the similar central wavelength in MODIS data is band 2 and band 6.Consequently NDBI $_{\text {MODIS }}$ is built from band 2 and band 6 based on MODIS data, as expressed in Eq. 2 .

$$
N D B I_{\text {ModIs }}=(B 6-B 2) /(B 6+B 2)
$$

IBI (Index-based Built-up Index) is constituted by NDBI, SAVI and MNDWI the theory of IBI is that the main land covers in urban is vegetation, built-up land and water body .NDVI is the vegetation, NDBI is the built-up land and MNDWI is the water body. The IBI is expressed in Eq.3. 


$$
I B I=\frac{[N D B I-(S A V I+M N D W I) / 2]}{[N D B I+(S A V I+M N D W I) / 2]}
$$

The IBI index expressed with band information based on TM image in Eq.4.

$$
I B I=\frac{2 T M 5 /(T M 4+T M 5)-[T M 4 /(T M 4+T M 3)+T M 2 /(T M 2+T M 5)]}{2 T M 5 /(T M 5+T M 4)+[T M 4 /[T M 4+T M 3]+T M 2 /(T M 2+T M 5)]}
$$

The equation is expressed with MODIS band information is in the Eq.5.

$$
I B I=\frac{2 B 6 /(B 6+B 2))-[B 2 /(B 2+B 1)+B 4 /(B 4+B 6)]}{2 B 6 /(B 6+B 2)+[B 2 /(B 2+B 1]+B 4 /(B 4+B 6)]}
$$

Where $\mathrm{B} 1, \mathrm{~B} 2, \mathrm{~B} 4$ and $\mathrm{B} 6$ are the band information of MODIS data, but in this paper build a new building index based on MOD09A1 product. The new building index is expressed in Eq.6.

$$
\begin{array}{r}
N B I=P_{(\text {urban })} \times \frac{\text { Band } 4-\text { Band } 1}{B a n d 4+B a n d 1} \\
P_{(\text {urban })}=D N_{D \text { MSP }} / D M S P_{\text {MAX }}
\end{array}
$$

Where $\mathrm{P}_{\text {(urban) }}$ is the percent of urban areas. The value of $\mathrm{DN}_{\mathrm{DMSP}}$ is provided by DMSP/OLS image, and DMSP $\mathrm{DAX}_{\mathrm{M}}$ is equal to 63.Using this model, a probabilities image of urban land cover was estimated in study region. The theory of NBI is that Built-up land value of band 4 is smaller than band 1 while other land covers has bigger $\mathrm{DN}$ value on band 4 than on band1. The high value of DMSP must be urban and the low value of DMSP image should be urban or county. The stable night light data is prior knowledge for new building index that extract regional urban area accurately and simply.

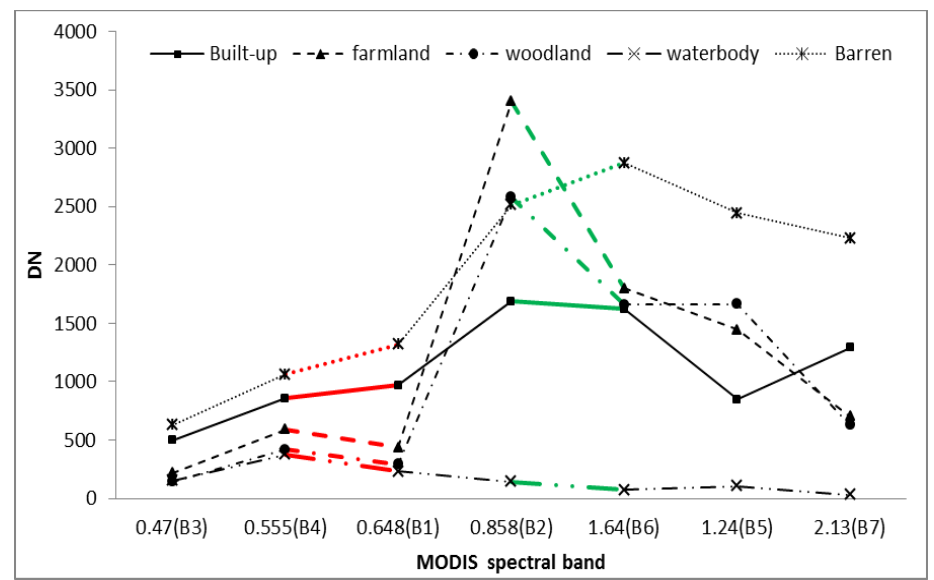

Fig. 2 spectral profiles of MODIS image (0.47, 0.555, 0.648, 0.858, 1.64, 1.24 and 2.13 are center wavelength of MODIS image $, B 1, B 2, B 3, B 4, B 5, B 6$ and $B 7$ are band information of MODIS image).

Figure2 show that Built-up land value of band 4 is smaller than band 1 while other land covers has bigger DN value on band 4 than on band1.the green part of curve is the basic band information of $\mathrm{NDBI}_{\mathrm{MODIS}}$.the red part of curve is the basic band information of NBI.

\section{VALIDATION OF NBI}

The NBI has been verified using the MODIS image of Beijing-tianjin-hebei, Yangtze River Delta and Pearl River Delta urban agglomeration in China (see figure 3), acquired on September 2010.
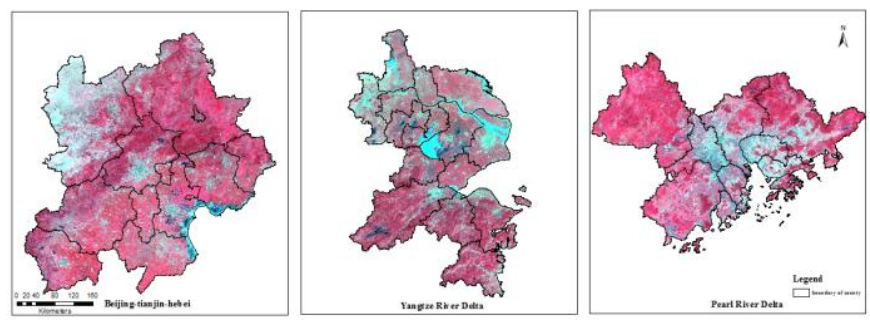

Fig. 3 MODIS image of Beijing-tianjin-hebei, Yangtze River Delta and Pearl River Delta urban agglomeration
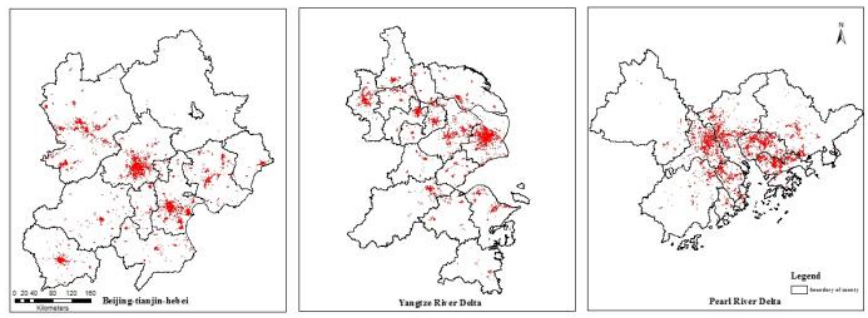

Fig. 4 the extraction results of NBI based on MODIS data.

The validation of the NBI is carried out by the confusion

\begin{tabular}{|c|c|c|}
\hline \multicolumn{3}{|c|}{$\begin{array}{l}\text { Tab.1 the accuracy assessment of extraction based on NBI in } \\
\text { Beijing-tianjin-hebei, Yangtze River Delta and Pearl River Delta } \\
\text { urban agglomeration }\end{array}$} \\
\hline Region & Overall Accuracy & Kappa \\
\hline Beijing-tianjin-hebei & $87.25 \%$ & 0.6537 \\
\hline Yangtze River Delta & $87.50 \%$ & 0.6713 \\
\hline Pearl River Delta & $90.50 \%$ & 0.8022 \\
\hline
\end{tabular}
matrix and Kappa coefficient assessment method of the accuracy for the extracted regional urban. The finer reference data is Google Earth image at $30 \mathrm{~m}$ spatial resolution.

Fig. 4 and Tab. 1 show that the accuracy of built -up land extraction based on NBI is rational. NBI has universality. The study area accuracy of regional urban extraction are all higher than $87 \%$ and Kappa are larger than 0.6.

\section{COMPARISON OF NBI WITH IBI AND NDBI}

NDBI,IBI and NBI have been compared using the MODIS image of Beijing-tianjin-hebei, Yangtze River Delta and Pearl River Delta urban agglomeration in ChinaThese building 
indexes are built using similar band information with TM image.The NBI, NDBI and IBI are compared based on pixel , area and all accuracy.

Firstly, the extraction result is compared from all acuracy. Fig.5show that NBI has the best result based on the comparision of NDBI,IBI and NBI.The extraction of NDBI is the worst result that the built-up land of Beijing-tianjin-hebei urban agglomeration almost is unbuilt.The building land of Yangtze River Delta and Pearl River Delta urban agglomeration are smaller than actural area.the extraction result of IBI is better than NDBI,but built-up land of extraction is also smaller than actural area.finally,the extraction result of NBI is the best.
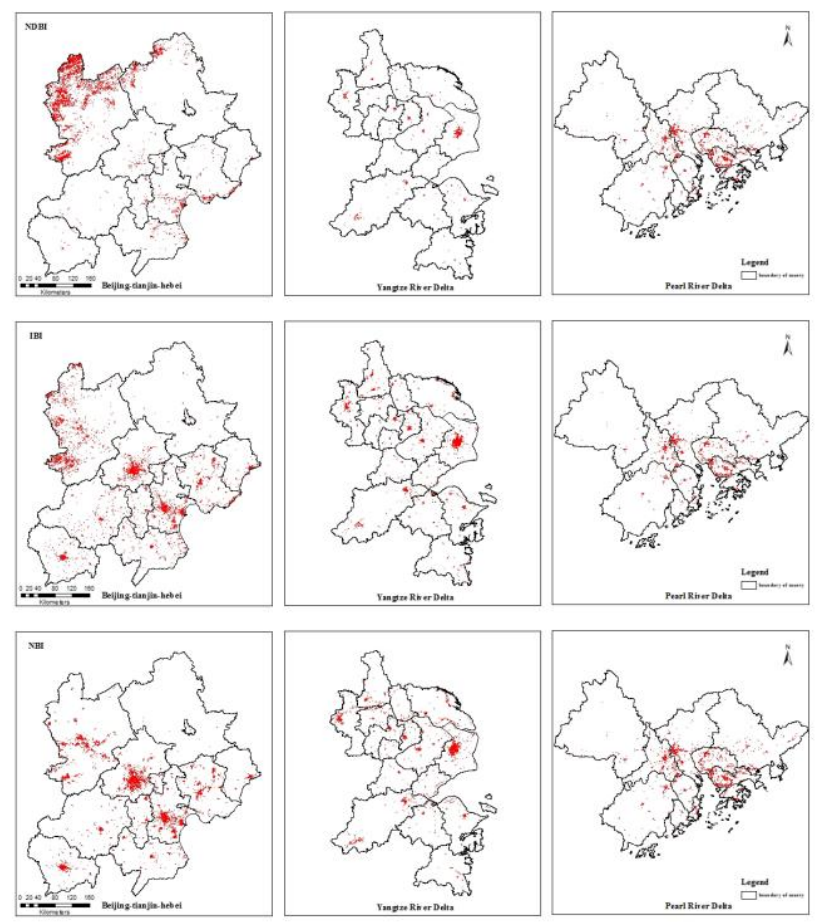

Fig. 5 the comparison of NDBI, IBI and NBI

\begin{tabular}{|c|c|c|c|}
\hline \multicolumn{4}{|c|}{$\begin{array}{l}\text { Tab.2 the accuracy assessment of extraction based on NDBI,IBI and NBI in } \\
\text { Beijing-tianjin-hebei, Yangtze River Delta and Pearl River Delta urban } \\
\text { agglomeration }\end{array}$} \\
\hline & Region & $\begin{array}{c}\text { Overall } \\
\text { Accuracy }\end{array}$ & Kappa \\
\hline \multirow{3}{*}{ NDBI } & Beijing-tianjin-hebei & $34 \%$ & 0.1023 \\
\hline & Yangtze River Delta & $75.25 \%$ & 0.5067 \\
\hline & Pearl River Delta & $72.50 \%$ & 0.4533 \\
\hline \multirow{3}{*}{ IBI } & Beijing-tianjin-hebei & $80 \%$ & 0.5321 \\
\hline & Yangtze River Delta & $80.25 \%$ & 0.5565 \\
\hline & Pearl River Delta & $84.50 \%$ & 0.6101 \\
\hline \multirow[t]{3}{*}{ NBI } & Beijing-tianjin-hebei & $87.25 \%$ & 0.6537 \\
\hline & Yangtze River Delta & $87.50 \%$ & 0.6713 \\
\hline & Pearl River Delta & $90.50 \%$ & 0.8022 \\
\hline
\end{tabular}

Tab. 2 show that NBI is the best result compared with NDBI and IBI.the extraction result of NBI in Beijing-tianjin-hebei, Yangtze River Delta and Pearl River Delta urban agglomeration are all larger than $85 \%$.the accuracy of result of NDBI and IBI are lesser than $85 \%$.the extraction of NDBI in Beijing-tianjin-hebei urban agglomeration even is lesser than $40 \%$.the NBI is the best accuracy compared with NDBI and IBI. The IBI also has a great accuracy,but the accuracy of IBI is lesser than NBI.

Fig.5 and Tab.2 show that the built-up land of NBI has the best result from all accuracy.Then the extraction result of NDBI,IBI and NBI are compared from pixel and area accuracy.
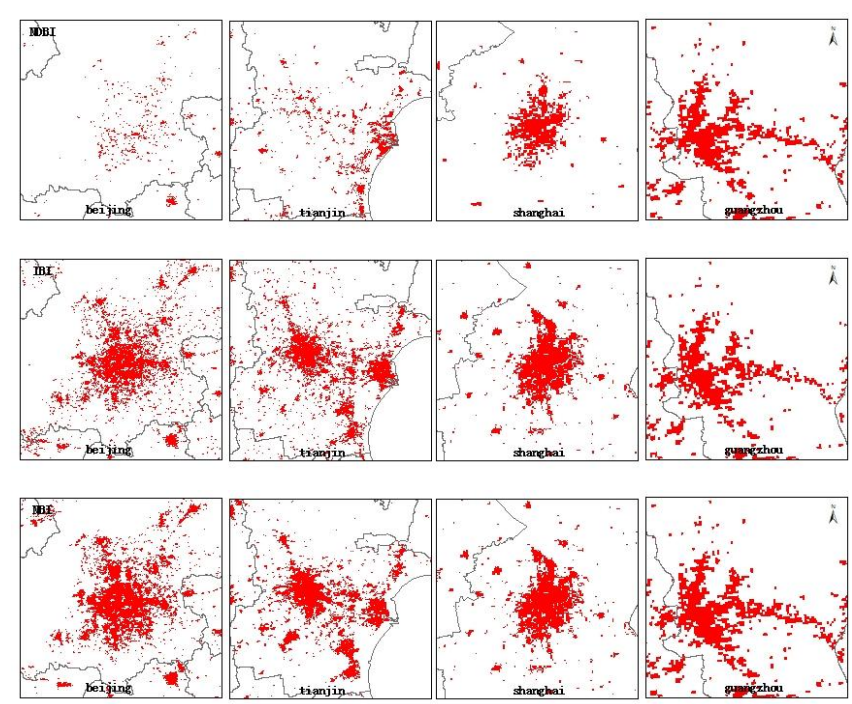

Fig. 6 the comparison of NDBI, IBI and NBI based on city level

The extraction result ofNDBI, IBI and NBI are compared from city and area level.Fig.6 show that the accuracy of built-up land extraction based on NBI in Beijing, Tianjin,and Guangzhou and shanghai cities is the best of all. The extraction result of NDBI in Beijing and Tianjin cities are worst. The extraction result of NDBI, IBI and NBI are compared based on area accuracy. The correlation between extraction and actualareais area accuracy. The extraction result of NBI in Beijing, Tianjin, Guangzhou and shanghai cities are the best of all. The built-up land of extraction based on NDBI is the worst of all. The IBI has similar result with NBI.

Fig.7and Fig.8 shows that Area accuracy is presented by the correlation between extraction and actual area. The area accuracy of NBI is the best of all. The correlation of NDBI is worse than IBI and NBI in Beijing-tianjin-hebei urban agglomeration. The extraction result of NDBI in Beijing and Tianjin cities is the worst of all. The correlation between actual and extraction of NBI in all four cities has a high value, and then the NBI has a high area accuracy. The NBI is the best of all. In this paper, NBI is better than NDBI and IBI. The extraction result of NBI has a great validation in regional urban. Area and all accuracy of NBIare best of all. 

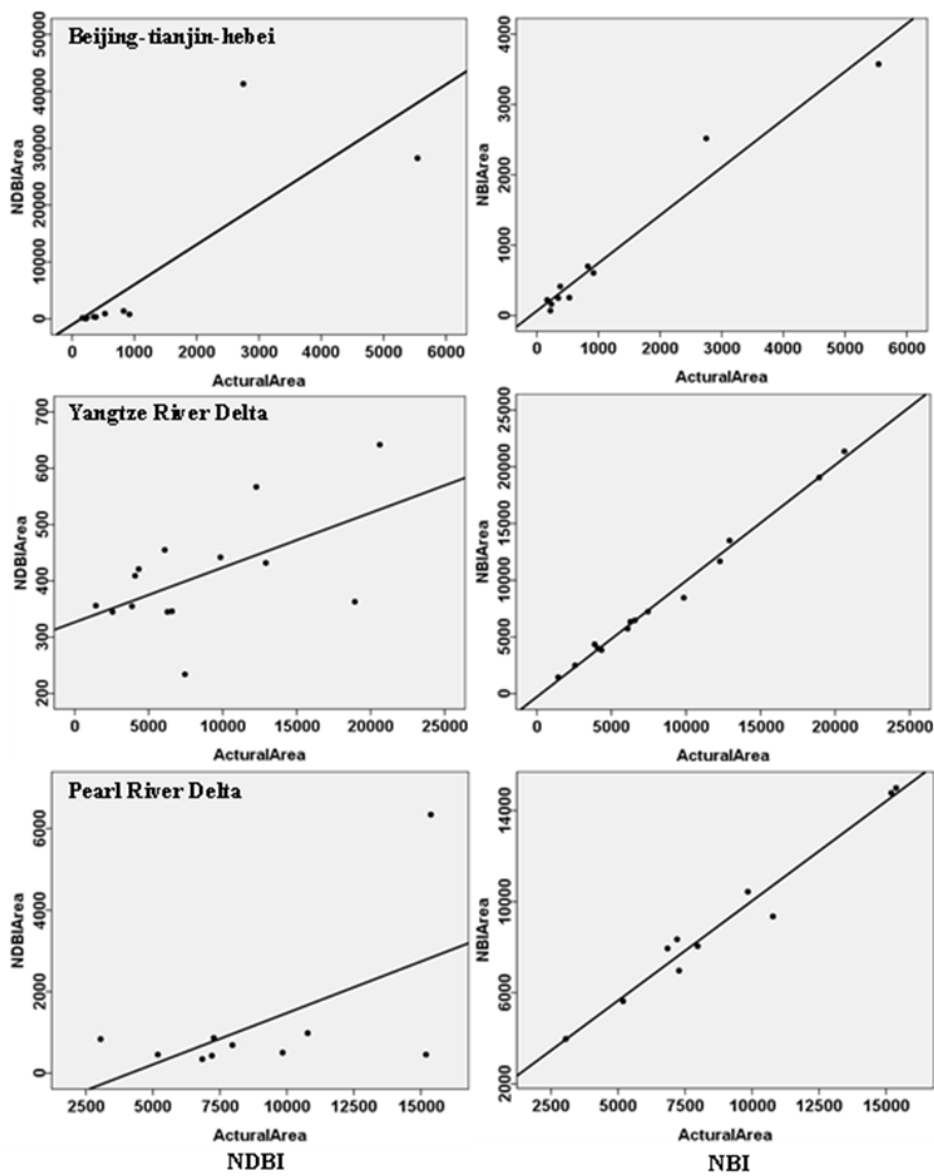

Fig. 7 the comparison of NDBI and NBI based on area accuracy
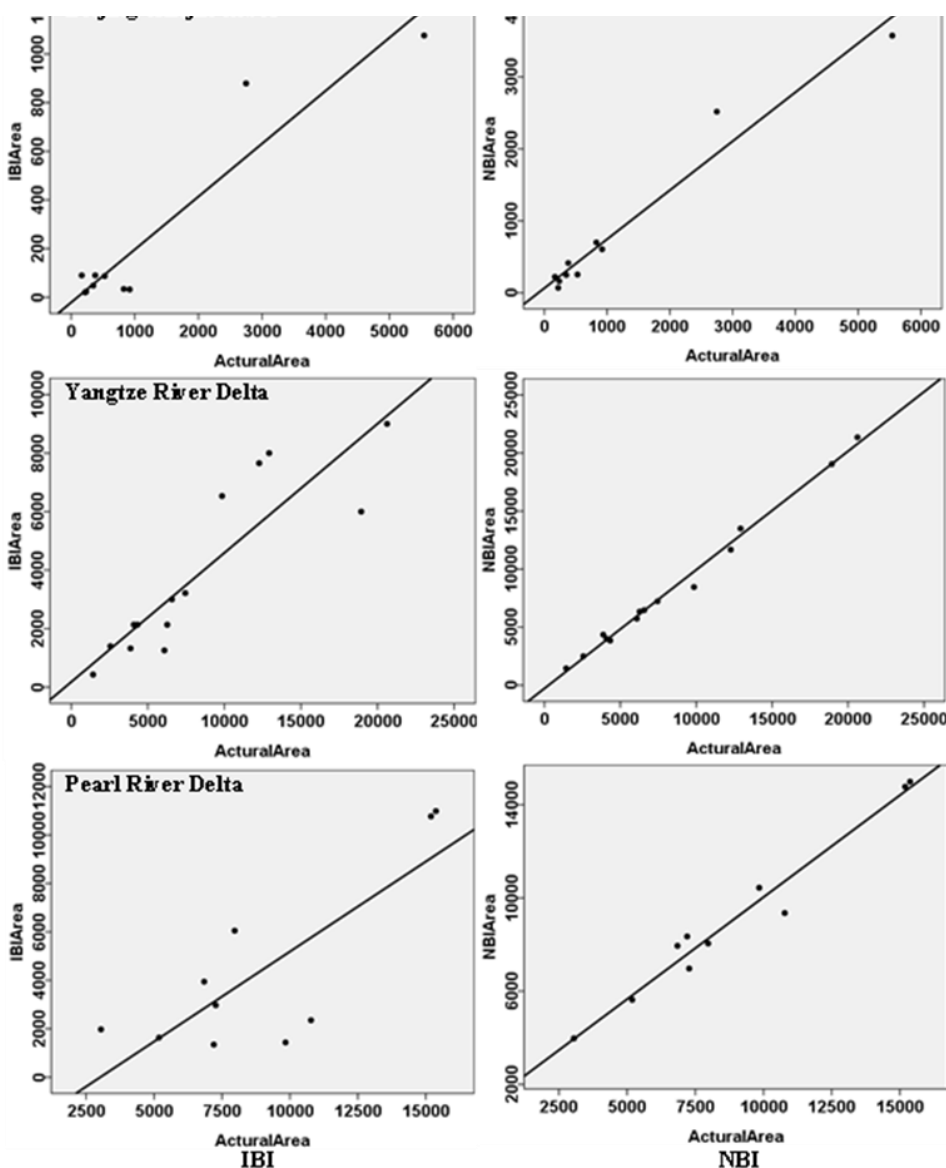

\section{CONCLUSION}

NBI is builtbased on the theory that Built-up land value of band 4 is smaller than band 1 while other land covers has bigger DN value on band 4 than on band1. The NBI had been verified using the MODIS image of Beijing-tianjin-hebei, Yangtze River Delta and Pearl River Delta urban agglomeration in China. The validation of NBI is great because of extraction accuracy in Beijing-tianjin-hebei, Yangtze River Delta and Pearl River Delta urban agglomeration are all larger than $85 \%$.

NDBI, IBI and NBI had been compared using the MODIS image of Beijing-tianjin-hebei, Yangtze River Delta and Pearl River Delta urban agglomeration in China. NDBI and IBI are built using similar band information with TM image. The NBI, NDBI and IBI are compared based on pixel, area and all accuracy.

Pixel and all accuracy are carried out by the confusion matrix and Kappa coefficient assessment method of the accuracy for the extracted regional urban. The finer reference data is Google Earth image at $30 \mathrm{~m}$ spatial resolution. The all and pixel accuracy of NBI is the best of all by comparing with NDBI and IBI.

Area accuracy is presented by the correlation between extraction and actual area. The area accuracy of NBI is the best of all. The correlation of NDBI is worse than IBI and NBI in Beijing-tianjin-hebei urban agglomeration. The extraction result of NDBI in Beijing and Tianjin cities is the worst of all. The correlation between actual and extraction of NBI in all four cities has a high value, and then the NBI has a high area accuracy. The NBI is the best of all.

NBI is better than NDBI and IBI.The extraction result of NBI has a great validation in regional urban. Area and all accuracy of NBI are best of all.

\section{REFERENCES}

[1] ALIMUJIANG K, RRYUTARO T, NGUYEN $\mathrm{T}$ H, et al. Global Urban Characterization using population density, DMSP and MODIS data [M]. 2009.

[2] SCHNEIDER A, FRIEDL M A, POTERE D. A new map of global urban extent from MODIS satellite data [J]. Environmental Research Letters, 2009, 4(4): 1-11.

[3] SCHNEIDER A, FRIEDL M A, POTERE D. Mapping global urban areas using MODIS 500-m data: New methods and datasets based on 'urban ecoregions' [J]. Remote Sensing of Environment, 2010, 114(8): 1733-1746.

[4] ZHA Y, GAO J, NI S. Use of normalized difference built-up index in automatically mapping urban areas from TM imagery [J]. International Journal of Remote Sensing, 2003, 24(3): 583-594.

[5] XU H. A new index for delineating built-up land features in satellite imagery [J]. International Journal of Remote Sensing, 2008, 29(14): 4269-4276.

[6] XU H Q. Extraction of urban built-up land features from Landsat imagery using a thematic-oriented index combination technique $[\mathrm{J}]$. Photogrammetric Engineering and Remote Sensing, 2007, 73(12): 1381-1391. 